\title{
BMJ Open Association between white blood cell count and non-alcoholic fatty liver disease in urban Han Chinese: a prospective cohort study
}

\author{
Shukang Wang, ${ }^{1}$ Chengqi Zhang, ${ }^{2}$ Guang Zhang, ${ }^{2}$ Zhongshang Yuan, ${ }^{1}$ \\ Yanxun Liu, ${ }^{1}$ Lijie Ding, ${ }^{1}$ Xiubin Sun, ${ }^{1}$ Hongying Jia, ${ }^{3}$ Fuzhong Xue ${ }^{1}$
}

To cite: Wang S, Zhang C, Zhang G, et al. Association between white blood cell count and non-alcoholic fatty liver disease in urban Han Chinese: a prospective cohort study. BMJ Open 2016;6: e010342. doi:10.1136/ bmjopen-2015-010342

- Prepublication history and additional material is available. To view please visit the journal (http://dx.doi.org/ 10.1136/bmjopen-2015010342).

Received 23 October 2015 Revised 23 March 2016 Accepted 18 April 2016

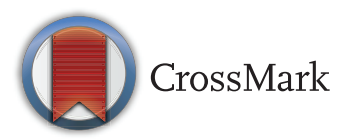

${ }^{1}$ Department of Biostatistics, School of Public Health, Shandong University, Jinan, China

${ }^{2}$ Health Management Center, Shandong Provincial QianFoShan Hospital, Jinan, China

${ }^{3}$ The Second Hospital of Shandong University, Jinan, China

Correspondence to Professor Fuzhong Xue; xuefzh@sdu.edu.cn

\section{ABSTRACT}

Objectives: The white blood cell (WBC) count is a simple and convenient marker of inflammation for use in medical practice; however, its association with nonalcoholic fatty liver disease (NAFLD) has not been determined. We examined the relationship between WBC and NAFLD to provide a convenient and useful marker for the prediction of NAFLD.

Setting: A longitudinal cohort participating in a large health check-up programme for the Chinese population was selected and followed up from 2005 to 2011.

Participants: A total of 21307 male and female participants without NAFLD who underwent health check-ups at least twice between 2005 and 2011 were included in this study. 15201 participants (7286 men and 7915 women) were eligible for inclusion.

Results: The baseline distribution of age, WBC, body mass index (BMI), systolic blood pressure (SBP), diastolic blood pressure (DBP), fasting plasma glucose (FPG), total cholesterol (TC), triglyceride (TG), highdensity lipoprotein cholesterol (HDL-C), low-density lipoprotein cholesterol (LDL-C), serum total protein (TP), albumin (ALB) and globin (GLO) and the prevalence of males, hypertension, hyperglycaemia, smoking and regular exercise were significantly different between the incident NAFLD and non-NAFLD groups $(p<0.05)$. Cox proportional hazards regression analysis was performed to estimate the HRs and $95 \%$ Cls of WBC, which predicted the occurrence of NAFLD. Compared with the lowest WBC quartile (Q1), the HRs and $95 \% \mathrm{Cls}$ of the other WBC quartiles (Q2, Q3 and Q4) for incident NAFLD were 1.090 (0.978 to 1.215 ), 1.174 (1.055 to 1.305 ) and 1.152 (1.035 to 1.281 ), respectively, after adjusting for age, gender, smoking, regular exercise, BMI, hypertension, hyperglycaemia, TC, TG, HDL-C, LDL-C, ALB and GLO.

Conclusions: Our study clearly showed that WBC count was a significant factor associated with incident NAFLD in Han Chinese.

\section{INTRODUCTION}

Non-alcoholic fatty liver disease (NAFLD) is a clinicopathological condition that ranges from simple steatosis to steatohepatitis,

\section{Strengths and limitations of this study}

- This was a longitudinal rather than a crosssectional study of a cohort selected from a large health check-up programme in a Chinese population and followed up from 2005 to 2011.

- An association between white blood cell (WBC) count and non-alcoholic fatty liver disease (NAFLD) was confirmed: WBC count can be regarded as an independent predictor of NAFLD.

- This finding cannot be easily extended to the general population, since the subjects were selected from a routine health check-up programme.

- Ultrasonography is inadequate for diagnosing NAFLD, and is less accurate than the gold standard biopsy.

- The severity of the NAFLD was not clearly defined in the health check-up data.

fibrosis or cirrhosis of the liver. ${ }^{1}$ It is a very common liver disease in Europe ${ }^{2}$ and the $\mathrm{USA}^{3}$ affecting more than $30 \%$ of the general population. ${ }^{4} 5$ The prevalence of NAFLD is also rising rapidly in Asia and China $^{6}{ }^{7}$ because of economic development and changes in lifestyle, having increased in China from less than $10 \%$ of the general population in China in the 1990s to approximately $20 \%$ in $2013 .{ }^{8}$

Studies have shown that NAFLD can progress to more severe liver diseases, such as hepatocellular carcinoma and liver failure, ${ }^{9} 10$ thus increasing liver-related mortality and morbidity in the population. ${ }^{11}$ Furthermore, it is associated with metabolic diseases ${ }^{12}$ and significantly increases the risk of cardiovascular disease $(\mathrm{CVD})^{13}$ and type 2 diabetes. ${ }^{14}$ NAFLD has become one of the most important public health problems worldwide. ${ }^{15}$

Various inflammatory markers, including C-reactive protein (CRP) ${ }^{16}$ interleukin-6 $(\mathrm{IL}-6),{ }^{17-19}$ tumour necrosis factor- $\alpha(\mathrm{TNF}-\alpha)^{20}$ 
and serum IgA level, ${ }^{21}$ have also been associated with NAFLD. However, to date, only one cross-sectional study with Korean adults has shown a significant association between white blood cell (WBC) count and NAFLD, ${ }^{22}$ and it is difficult to determine the temporal relationship between an elevated WBC count and the incidence of NAFLD. Cohort studies should be conducted to confirm the temporal relationship between WBC level and incident NAFLD. Moreover, the WBC count is a simple, readily available and inexpensive marker of inflammation for use in medical practice, and has become an important predictor of infectious diseases and of CVD, diabetes $^{23}$ and metabolic syndrome (MS). ${ }^{24}$ We therefore conducted a large-scale health assessment-based longitudinal cohort study in an urban Han Chinese population to determine the relationship between WBC count and NAFLD. The findings from such a study may indicate a convenient and useful marker for the further risk appraisal of NAFLD.

\section{MATERIALS AND METHODS}

\section{Study population and cohort design}

The subjects in our longitudinal cohort were selected from a routine health check-up system based in the Center for Health Management of Shandong Provincial QianFoShan Hospital and Shandong Provincial Hospital. A total of 21307 male and female participants without NAFLD who visited the health check-up system at least twice between 2005 and 2011 were included. Questions about alcohol intake included the type of alcohol consumed, the frequency of alcohol consumption per week and the usual amount per day $(\geq 20 \mathrm{~g}$ / day). Alcohol intake was further coded as an ordered categorical variable as follows: 0 , never; 1 , seldom; 2, often, wine; 3, often, beer; 4, often, Chinese spirits; and 5 , often, mixed/all types. Persons with a value above 1 were considered regular alcohol users. Subjects with regular alcohol intake $(\mathrm{n}=4774)$ were excluded as were 538 subjects with a positive serological marker for hepatitis B surface antigen ( $\mathrm{HBsAg})$, hepatitis $\mathrm{C}$ virus antibody (HCVAb) or a history of chronic liver disease. Additionally, subjects with WBC counts $<4.00$ or $>10.00$ cells $10^{9} / \mathrm{L}$ were excluded to eliminate any patients with severe infection. Because some participants may have met more than one exclusion criterion, the total number of participants who were eligible for this study was 15201 . This study was approved by the Ethics Committee of the School of Public Health, Shandong University. Written informed consent was obtained from all participants.

\section{Investigation and measurements}

During baseline investigations and follow-up, trained interviewers administered a standardised questionnaire to obtain information on age, sex, smoking status and regular exercise. After an overnight fast of at least 12 hours, all subjects underwent a standardised medical examination that included routine anthropometric, clinical and laboratory tests. The anthropometric measurements involved height, weight and blood pressure (BP). Two BP values were taken 5-15 min apart on the right arm by trained examiners. Height and weight were measured after the participants had removed their shoes, heavy clothing and belts. Body mass index (BMI) was calculated as weight $(\mathrm{kg}) /$ height $^{2}(\mathrm{~m})$ and was used as an estimate of obesity. Laboratory tests included triglycerides (TG), total cholesterol (TC), low-density lipoprotein cholesterol (LDL-C), high-density lipoprotein cholesterol (HDL-C), serum total protein (TP), albumin (ALB) and globin (GLO). All participants also underwent abdominal B-mode ultrasonography.

\section{Definitions of NAFLD, hypertension and hyperglycaemia}

In accordance with the revised definition and treatment guidelines for NAFLD issued by the Chinese Hepatology Association in February 2006, ${ }^{25}$ NAFLD was diagnosed by abdominal ultrasonography that revealed a bright liver and a diffusely echogenic change in the liver parenchyma. Participants diagnosed with alcoholic fatty liver disease, infectious viral hepatitis (HBsAg or HCVAb positive) and other causes of steatosis were excluded. The presence of hypertension was defined as SBP $\geq 140 \mathrm{~mm}$ $\mathrm{Hg}$ and diastolic BP (DBP) $\geq 90 \mathrm{~mm} \mathrm{Hg}$, or had SBP $\geq 140 \mathrm{~mm} \mathrm{Hg}$ or diastolic BP (DBP) $\geq 90 \mathrm{~mm} \mathrm{Hg}$ or a previous diagnosis. The presence of hyperglycaemia was defined as fasting plasma glucose (FPG) $\geq 6.1 \mathrm{mmol} / \mathrm{L}$ or 2-hour post-prandial glucose $(\mathrm{PG}) \geq 7.8 \mathrm{mmol} / \mathrm{L}$ or a previous diagnosis.

\section{Statistical analysis}

Multiple imputations were performed to account for missing values. Because the imputation method depends on the patterns of the missing data and the types of the imputed variables, without loss of generality, the Markov chain Monte Carlo (MCMC) method provided by the MI procedure of SAS was used. ${ }^{26}$ Most variables had less than $2 \%$ missing observations before imputation, except for smoking and physical activity which had less than $10 \%$ missing values.

Descriptive statistics were generated for all baseline characteristics according to gender and incident NAFLD or no incident NAFLD. Continuous variables were expressed as the mean and SD (mean $\pm \mathrm{SD})$, or median (quartile range) based on whether their distribution was normal or skewed as judged by histogram. A two-sample t-test and Wilcoxon rank sum test were used to determine the group difference. Categorical variables were expressed as percentages, and $\chi^{2}$ tests were used to determine the difference for categorical data. Additionally, WBC (cells $10^{9} / \mathrm{L}$ ) were further categorised separately into quartiles: $\mathrm{Q} 1$ : $\mathrm{WBC}<5.35$, Q2: $5.35 \leq \mathrm{WBC}<6.20$, Q3: $6.20 \leq \mathrm{WBC}<7.14$, and $\mathrm{Q} 4: \mathrm{WBC} \geq 7.14$. The $F$ test or Kruskal-Wallis test was used to compare differences between WBC quartiles. LSD tests were used for multiple comparisons of continuous variables, and the Bonferroni 
Table 1 Baseline characteristics according to gender

\begin{tabular}{|c|c|c|c|}
\hline Characteristics & Males $(n=7286)$ & Females $(n=7915)$ & Total $(n=15201)$ \\
\hline Age & $45.49 \pm 16.49$ & $40.60 \pm 13.00^{*}$ & $42.95 \pm 14.98$ \\
\hline WBC count $\left(10^{9} / \mathrm{L}\right)$ & $6.47 \pm 1.28$ & $6.19 \pm 1.25^{\star}$ & $6.32 \pm 1.28$ \\
\hline BMI $\left(\mathrm{kg} / \mathrm{m}^{2}\right)$ & $24.33 \pm 2.92$ & $22.80 \pm 3.19^{\star}$ & $23.53 \pm 3.12$ \\
\hline $\mathrm{SBP}(\mathrm{mm} \mathrm{Hg})$ & $126.68 \pm 18.20$ & $115.74 \pm 17.96^{*}$ & $120.98 \pm 18.88$ \\
\hline DBP (mm Hg) & $73.77 \pm 10.90$ & $69.20 \pm 10.48^{\star}$ & $71.39 \pm 10.93$ \\
\hline FPG (mmol/L) & $5.13 \pm 1.06$ & $4.89 \pm 0.83^{\star}$ & $5.01 \pm 0.95$ \\
\hline $\mathrm{TC}(\mathrm{mmol} / \mathrm{L})$ & $4.90 \pm 0.90$ & $4.89 \pm 0.97$ & $4.90 \pm 0.94$ \\
\hline $\mathrm{TG}(\mathrm{mmol} / \mathrm{L})$ & $1.16(0.90)$ & $0.88(0.71)^{\star}$ & $1.01(0.83)$ \\
\hline HDL-C (mmol/L) & $1.25(0.39)$ & $1.45(0.43)^{\star}$ & $1.35(0.44)$ \\
\hline $\mathrm{TP}(\mathrm{g} / \mathrm{L})$ & $73.75 \pm 4.35$ & $73.91 \pm 4.25^{\star}$ & $73.83 \pm 4.30$ \\
\hline ALB (g/L) & $46.80 \pm 2.53$ & $45.93 \pm 2.49^{\star}$ & $46.35 \pm 2.54$ \\
\hline GLO (g/L) & $26.95 \pm 3.98$ & $27.97 \pm 3.95^{\star}$ & $27.48 \pm 4.00$ \\
\hline LDL-C (mmol/L) & $2.85 \pm 0.71$ & $2.70 \pm 0.74^{\star}$ & $2.77 \pm 0.73$ \\
\hline Hypertension (\%) & $1892(26.0)$ & $979(12.4)^{\star}$ & 2871 (18.9) \\
\hline Hyperglycaemia (\%) & $720(9.9)$ & $390(4.9)^{\star}$ & $1110(7.3)$ \\
\hline Current smoker (\%) & $2153(29.5)$ & $36(0.5)^{\star}$ & $2189(14.4)$ \\
\hline Regular exercise (\%) & $2953(40.5)$ & $1759(22.2)^{\star}$ & $4712(31)$ \\
\hline
\end{tabular}

correction was used for multiple comparisons of categorical variables. Cox proportional hazards regression analysis was performed to estimate the HRs and $95 \%$ CIs of WBC, which predicted the occurrence of NAFLD. We constructed three Cox proportional hazards regression models by adjusting different confounding factors, including age, gender, smoking, regular exercise, BMI, hypertension, hyperglycaemia, TC, TG, HDL-C, LDL-C, ALB and GLO at baseline. SAS V.9.1.3 (SAS Institute, Cary, North Carolina, USA) and SPSS (V.20.0) were used to perform all statistical analyses.

\section{RESULTS}

A total of 15201 subjects (7286 men and 7915 women) were included in this study. The baseline distributions of age, WBC, BMI, SBP, DBP, FPG, TC, TG, HDL-C, LDL-C, TP, ALB and GLO by gender; the prevalence of hypertension, hyperglycaemia, smoking and regular exercise; and comparisons between males and females are summarised in table 1 . The mean age was $45.49 \pm 16.49$ years in males and $40.60 \pm 13.00$ years in females. All other variables except TC were significantly different between males and females $(\mathrm{p}<0.05)$.

The baseline characteristics of the subjects according to WBC quartile are shown in table 2. The mean values of age, BMI, SBP, DBP, FPG, TC, TG, LDL-C, TP and GLO and the prevalence of hypertension, hyperglycaemia and smoking were highest in the fourth WBC quartile, in contrast to HDL-cholesterol which was lowest in the fourth WBC quartile. Significant differences were found for the other characteristics except for regular exercise and ALB among the different WBC quartiles $(p<0.05)$. Significant differences were found between various WBC quartiles for BMI, SBP, DBP, FPG, TG, HDL-C, LDL-C, TP, GLO and hypertension $(\mathrm{p}<0.05)$. Significant differences for age and TC were found between Q1 and Q3 or Q4, and between $\mathrm{Q}^{2}$ and $\mathrm{Q}^{4}$. Significant differences for hyperglycaemia and smoking were found between Q1 and Q2, Q3 or Q4, and between Q2 and Q4.

Table 3 shows the baseline distributions of age, WBC, BMI, SBP, DBP, FPG, TC, TG, HDL-C, LDL-C, TP, ALB and GLO and the prevalence of males, hypertension, hyperglycaemia, smoking and regular exercise according to NAFLD status during follow-up. All variables were significantly different between incident NAFLD and non-NAFLD groups, and the averages of the other characteristics in the NAFLD group (except HDL-C and ALB) were significantly higher than those in the non-NAFLD group $(\mathrm{p}<0.05)$. Online supplementary table $\mathrm{S} 1$ shows the baseline characteristics according to NAFLD status and are grouped by gender.

Table 4 shows the HRs (95\% CIs) of WBC as the independent variable in different Cox proportional hazards models. In the unadjusted model, compared with the lowest WBC quartile (Q1), the HRs and 95\% CIs of the other WBC quartiles (Q2, Q3 and Q4) in the NAFLD group were $1.310(1.180$ to 1.454$), 1.511$ (1.365 to 1.673 ) and 1.654 (1.497 to 1.828$)$, respectively. In the first model, after adjusting for age and gender, compared with the lowest WBC quartile (Q1), the HRs and 95\% CIs of the other WBC quartiles (Q2, Q3 and Q4) in the NAFLD group were 1.241 (1.118 to 1.378$), 1.427$ (1.289 to 1.580 ) and 1.507 (1.363 to 1.666$)$, respectively, and age and gender were significant variables. In the second model, after adjusting for age, gender, smoking and regular exercise, compared with the lowest WBC 
Table 2 Comparison of baseline characteristics according to WBC quartile

\begin{tabular}{|c|c|c|c|c|c|}
\hline \multirow[b]{2}{*}{ Characteristics } & \multicolumn{5}{|l|}{ WBC quartiles } \\
\hline & Q1 & Q2 & Q3 & Q4 & p Value \\
\hline N (\%) & 3769 & 3825 & 3809 & 3798 & \\
\hline Age & $42.33 \pm 14.34$ & $42.79 \pm 15.02$ & $43.12 \pm 14.86$ & $43.54 \pm 15.64$ & 0.004 \\
\hline BMI $\left(\mathrm{kg} / \mathrm{m}^{2}\right)$ & $22.78 \pm 2.89$ & $23.35 \pm 3.10$ & $23.81 \pm 3.11$ & $24.18 \pm 3.19$ & $<0.001$ \\
\hline $\mathrm{SBP}(\mathrm{mm} \mathrm{Hg})$ & $117.73 \pm 17.48$ & $120.44 \pm 18.64$ & $121.86 \pm 18.94$ & $123.88 \pm 19.86$ & $<0.001$ \\
\hline DBP (mm Hg) & $69.49 \pm 10.46$ & $71.13 \pm 10.75$ & $72.00 \pm 10.94$ & $72.92 \pm 11.25$ & $<0.001$ \\
\hline FPG (mmol/L) & $4.91 \pm 0.81$ & $4.98 \pm 0.93$ & $5.04 \pm 0.98$ & $5.10 \pm 1.06$ & $<0.001$ \\
\hline $\mathrm{TC}(\mathrm{mmol} / \mathrm{L})$ & $4.84 \pm 0.94$ & $4.88 \pm 0.94$ & $4.92 \pm 0.93$ & $4.94 \pm 0.94$ & $<0.001$ \\
\hline $\mathrm{TG}(\mathrm{mmol} / \mathrm{L})$ & $0.86(0.67)$ & $0.97(0.80)$ & $1.06(0.82)$ & $1.19(0.94)$ & $<0.001$ \\
\hline HDL-C (mmol/L) & $1.41(0.43)$ & $1.36(0.44)$ & $1.33(0.43)$ & $1.29(0.43)$ & $<0.001$ \\
\hline LDL-C (mmol/L) & $2.69 \pm 0.72$ & $2.76 \pm 0.73$ & $2.80 \pm 0.72$ & $2.85 \pm 0.73$ & $<0.001$ \\
\hline $\mathrm{TP}(\mathrm{g} / \mathrm{L})$ & $73.38 \pm 4.24$ & $73.74 \pm 4.23$ & $73.96 \pm 4.29$ & $74.25 \pm 4.38$ & $<0.001$ \\
\hline ALB (g/L) & $46.28 \pm 2.54$ & $46.36 \pm 2.56$ & $46.38 \pm 2.46$ & $46.38 \pm 2.62$ & 0.287 \\
\hline GLO (g/L) & $27.10 \pm 3.92$ & $27.38 \pm 4.01$ & $27.58 \pm 4.01$ & $27.87 \pm 4.01$ & $<0.001$ \\
\hline Hypertension (\%) & $539(14.3)$ & $683(17.9)$ & 772 (20.3) & 877 (23.1) & $<0.001$ \\
\hline Hyperglycaemia (\%) & $197(5.2)$ & $263(6.9)$ & $298(7.8)$ & $352(9.3)$ & $<0.001$ \\
\hline Current smoker (\%) & $372(9.9)$ & $481(12.6)$ & $555(14.6)$ & 781 (20.6) & $<0.001$ \\
\hline Regular exercise (\%) & 1169 (31.0) & 1194 (31.2) & 1197 (31.4) & $1152(30.3)$ & 0.839 \\
\hline
\end{tabular}

Q1: WBC <5.35; Q2: $5.35 \leq$ WBC <6.20; Q3: $6.20 \leq$ WBC $<7.14$; Q4: WBC $\geq 7.14$ cells $\times 10^{9} /$ L.

ALB, albumin; BMI, body mass index; DBP, diastolic blood pressure; FPG, fasting plasma glucose; GLO, globin; HDL-C, high-density lipoprotein cholesterol; LDL-C, low-density lipoprotein cholesterol; SBP, systolic blood pressure; TC, total cholesterol; TG, triglyceride; TP, serum total protein; WBC, white blood cell count.

quartile (Q1), the HRs and $95 \%$ CIs of the other WBC quartiles (Q2, Q3 and Q4) in the NAFLD group were 1.243 (1.116 to 1.384 ), 1.440 (1.296 to 1.600 ) and 1.558 (1.404 to 1.729$)$, respectively, and age, gender and regular exercise (not smoking) were significant variables. In the third model, after adjusting for age, gender, smoking, regular exercise, BMI, hypertension, hyperglycaemia, TC, TG, HDL-C, LDL-C, ALB and GLO compared with the lowest WBC quartile (Q1), the HRs and 95\% CIs of the other WBC quartiles (Q2, Q3 and Q4) in the NAFLD group were 1.090 (0.978 to 1.215$), 1.174$ (1.055 to 1.305 ) and 1.152 (1.035 to 1.281 ), respectively, and age, gender, regular exercise, BMI, hypertension, TG, HDL-C, LDL-C and GLO (not smoking, hyperglycaemia, TC or ALB) were significant variables.

Recently, Das et $a l^{27}$ has explored the association between haematological parameters and NAFLD. Our health check-up data showed that apart from WBC, that red blood cell count, haemoglobin, mean corpuscular volume, mean corpuscular haemoglobin concentration, red cell distribution width-SD (RDW-SD), neutrophil count and platelet count are also significantly associated with NAFLD (see online supplementary table S2).

\section{DISCUSSION}

The effects of NAFLD were not limited to the liver but damaged various body systems; the extrahepatic damage was serious and increased the risk of CVD and diabetes. ${ }^{28}$ Consequently, it is very important to predict NAFLD and determine its risk factors. Some studies have reported that age is associated with NAFLD prevalence and that prevalence increases with age, ${ }^{29} 30$ as also seen in our study. The results in our study also show that NAFLD incidence is higher in males than in females (table 3), which was similar to previous studies. ${ }^{31-33}$ Lifestyle factors ${ }^{34} 35$ associated with NAFLD included smoking and physical activity. The baseline rate of regular exercise in the NAFLD group was significantly higher than that in the non-NAFLD group $(p<0.001)$. This counter-intuitive finding may be partly due to the fact that other potential confounding factors (age, gender, BMI, hypertension, TG, HDL-C, LDL-C, GLO and smoking) were not adjusted for. However, the subsequent multivariate analysis showed regular exercise is indeed a protective factor against NAFLD. Additionally, and more importantly, a relationship between NAFLD and MS has been proposed in many studies which revealed that components of MS such as obesity, hypertension, dyslipidaemia and hyperglycaemia were independently associated with NAFLD. ${ }^{7} 3637$ The results in our study also demonstrated that MS components were significantly different between individuals in the NAFLD and non-NAFLD groups (table 3), suggesting that NAFLD may be the liver manifestation of MS. ${ }^{38} 39$ Therefore, we explored the relationship between WBC and NAFLD after adjusting for age, gender, lifestyle factors and MS components.

To our knowledge, only one cross-sectional study with Korean adults has shown a significant association between WBC count and NAFLD, ${ }^{22}$ which is consistent with our findings. Compared with the prospective cohort study, cross-sectional studies could not confirm the temporal association between WBC level and 
Table 3 Baseline characteristics according to NAFLD status

\begin{tabular}{|c|c|c|}
\hline Characteristics & $\begin{array}{l}\text { NAFLD } \\
(n=3376)\end{array}$ & $\begin{array}{l}\text { Non-NAFLD } \\
(n=11825)\end{array}$ \\
\hline Age & $48.89 \pm 15.45$ & $41.25 \pm 14.40^{*}$ \\
\hline Male (\%) & 2070 (61.3) & $5216(44.1)^{\star}$ \\
\hline $\begin{array}{l}\text { WBC count } \\
\left.\text { (cells } \times 10^{9} / \mathrm{L}\right)\end{array}$ & $6.53 \pm 1.27$ & $6.26 \pm 1.27^{\star}$ \\
\hline BMI $\left(\mathrm{kg} / \mathrm{m}^{2}\right)$ & $25.19 \pm 2.99$ & $23.06 \pm 2.99^{\star}$ \\
\hline $\mathrm{SBP}(\mathrm{mm} \mathrm{Hg})$ & $\begin{array}{l}128.80 \\
\pm 19.73\end{array}$ & $118.75 \pm 18.02^{*}$ \\
\hline DBP (mm Hg) & $75.10 \pm 11.28$ & $70.33 \pm 10.59^{*}$ \\
\hline FPG (mmol/L) & $5.22 \pm 1.03$ & $4.95 \pm 0.92^{*}$ \\
\hline $\mathrm{TC}(\mathrm{mmol} / \mathrm{L})$ & $5.14 \pm 0.97$ & $4.83 \pm 0.92^{*}$ \\
\hline $\mathrm{TG}(\mathrm{mmol} / \mathrm{L})$ & $1.31(0.97)$ & $0.93(0.74)^{\star}$ \\
\hline HDL-C (mmol/L) & $1.29(0.43)$ & $1.36(0.43)^{\star}$ \\
\hline LDL-C (mmol/L) & $2.98 \pm 0.74$ & $2.71 \pm 0.71^{\star}$ \\
\hline $\mathrm{TP}(\mathrm{g} / \mathrm{L})$ & $74.09 \pm 4.32$ & $73.76 \pm 4.29^{\star}$ \\
\hline ALB (g/L) & $46.18 \pm 2.57$ & $46.40 \pm 2.53^{*}$ \\
\hline GLO (g/L) & $27.90 \pm 4.09$ & $27.36 \pm 3.96^{\star}$ \\
\hline Hypertension (\%) & 1154 (34.2) & $1717(14.5)^{*}$ \\
\hline Hyperglycaemia (\%) & $391(11.6)$ & $719(6.1)^{\star}$ \\
\hline Current smoker (\%) & $634(18.8)$ & $1555(13.2)^{*}$ \\
\hline Regular exercise (\%) & $1286(38.1)$ & $3426(29.0)^{\star}$ \\
\hline \multicolumn{3}{|c|}{$\begin{array}{l}{ }^{*} \mathrm{p}<0.05 \text {, comparison between NAFLD and non-NAFLD. } \\
\text { ALB, albumin; BMI, body mass index; DBP, diastolic blood } \\
\text { pressure; FPG, fasting plasma glucose; GLO, globin; HDL-C, } \\
\text { high-density lipoprotein cholesterol; LDL-C, low-density lipoprotein } \\
\text { cholesterol; NAFLD, non-alcoholic fatty liver disease; SBP, systolic } \\
\text { blood pressure; TC, total cholesterol; TG, triglyceride; TP, serum } \\
\text { total protein; WBC, white blood cell count. }\end{array}$} \\
\hline
\end{tabular}

incident NAFLD. The results of cross-sectional studies can only provide markers for disease screening, not for disease prediction. WBC levels may not be a predictive factor for NAFLD unless confirmed in a cohort study.
In this cohort study, we confirmed a relationship between WBC count and NAFLD incidence in urban Han Chinese (table 4), even if WBC changes were within the normal range. The relationship between WBC count and NAFLD remained valid after adjusting for other relevant factors including age, gender, smoking, regular exercise, BMI, hypertension, hyperglycaemia, TC, TG, HDL-C, LDL-C, ALB and GLO. The results prove that an elevated WBC level is related to NAFLD incidence, a finding which provides novel and powerful evidence for a significant relationship between WBC level and NAFLD. Thus, WBC count may be used as an independent predictor of NAFLD and is expected to improve prediction power when combined with other risk factors such as age, gender, BMI and hypertension status. The importance of WBC in subjects with only NAFLD may be different from that in those with NAFLD, cirrhosis and non-alcoholic steatohepatitis (NASH). Further study is required to elucidate these relationships. Moreover, some liver function test markers such as alanine aminotransferase (ALT), aspartate aminotransferase (AST) and $\gamma$-glutamyl transferase (GGT) should be explored in further study.

This study suggests that inflammation plays an important role in the occurrence of NAFLD. Specifically, hepatic steatosis was the result of systemic inflammation, which is consistent with previous research. Many earlier studies have shown a significant association between CRP and NAFLD in Chilean Hispanic subjects, ${ }^{40}$ Asian Indians in North India, ${ }^{41}$ and Japanese ${ }^{42}$ and Korean individuals. ${ }^{43}$ These studies reported that elevated CRP was independently associated with the presence of NAFLD. Additionally, it was reported that serum TNF- $\alpha$, IL-6 and interleukin-8 (IL-8) levels were higher in people with NAFLD than in normal subjects, ${ }^{44}$

Table 4 HRs and their 95\% Cls from the Cox model for prediction of NAFLD using WBC as the independent variable

\begin{tabular}{|c|c|c|c|c|}
\hline & Unadjusted & Model 1* & Model 2† & Model 3‡ \\
\hline \multicolumn{5}{|c|}{ WBC count (cells $\left.\times 10^{9} / \mathrm{L}\right)$} \\
\hline$<5.35$ & Reference & Reference & Reference & Reference \\
\hline $5.35-6.20$ & $1.310(1.180$ to 1.454$)$ & 1.241 (1.118 to 1.378$)$ & $1.243(1.116$ to 1.384$)$ & $1.090(0.978$ to 1.215$)$ \\
\hline $6.20-7.14$ & $1.511(1.365$ to 1.673$)$ & $1.427(1.289$ to 1.580$)$ & $1.440(1.296$ to 1.600$)$ & $1.174(1.055$ to 1.305$)$ \\
\hline$\geq 7.14$ & $1.654(1.497$ to 1.828$)$ & $1.507(1.363$ to 1.666$)$ & $1.558(1.404$ to 1.729$)$ & $1.152(1.035$ to 1.281$)$ \\
\hline Age & & $1.019(1.017$ to 1.021$)$ & $1.019(1.017$ to 1.021$)$ & $1.007(1.005$ to 1.010$)$ \\
\hline \multicolumn{5}{|l|}{ Gender } \\
\hline Male & & Reference & Reference & Reference \\
\hline Female & & $0.618(0.576$ to 0.664$)$ & $0.607(0.563$ to 0.654$)$ & $0.746(0.689$ to 0.807$)$ \\
\hline Regular exercise & & & $0.922(0.855$ to 0.995$)$ & 0.917 (0.850 to 0.989$)$ \\
\hline BMI & & & & $1.153(1.138$ to 1.167$)$ \\
\hline Hypertension & & & & $1.230(1.126$ to 1.344$)$ \\
\hline TG & & & & $1.190(1.165$ to 1.216$)$ \\
\hline HDL-C & & & & 0.798 (0.708 to 0.899$)$ \\
\hline LDL-C & & & & $1.192(1.134$ to 1.253$)$ \\
\hline GLO & & & & $1.011(1.002$ to 1.020$)$ \\
\hline
\end{tabular}

${ }^{*}$ Adjusted by baseline age and gender.

†Adjusted by baseline age, gender, smoking and regular exercise.

$\ddagger$ Adjusted by baseline age, gender, smoking, regular exercise, BMI, hypertension, hyperglycaemia, TC, TG, HDL-C, LDL-C, ALB and GLO. BMI, body mass index; GLO, globin; HDL-C, high-density lipoprotein cholesterol; LDL-C, low-density lipoprotein cholesterol; NAFLD, non-alcoholic fatty liver disease; TG, triglyceride; WBC, white blood cell count. 
indicating that these inflammatory factors are associated with the development of NAFLD. However, WBC is more suitable than inflammatory factors because it is a simple, readily available and inexpensive marker of inflammation for use in medical practice.

There are two potential mechanisms for WBC participation in the onset of NAFLD. ${ }^{46}$ The first is insulin resistance which is the key factor associated with NAFLD. Insulin resistance causes TG synthesis and transport function disorders, and is a key mechanism in the development of MS. Indeed, NAFLD may be the liver manifestation of MS, ${ }^{9} 3947$ as a relationship between WBC count and MS components has been documented in previous studies. ${ }^{48} 49$ This study showed significant changes in the components of MS with increasing WBC quartiles (table 2), suggesting insulin resistance may link WBC count and NAFLD. The second is the fact that hepatocytes with too much lipid are vulnerable to injurious processes such as cytokines and oxidative stress, which lead to inflammation and liver fibrosis. $^{50} 51$ WBC count was frequently used to assess inflammatory status ${ }^{52}$ and so was associated with NAFLD.

Our study has several limitations. First, generalisability to the general population is unclear as the subjects in this study were recruited from a routine health check-up programme in an urban Han Chinese population in Shandong province. Consequently, further studies should be conducted to verify the relationship between WBC level and NAFLD in the general population. Second, the presence of NAFLD was assessed by experienced radiologists using abdominal ultrasonography, but we have no information on the intra- or inter-observer reliability of ultrasonographic examination. The diagnosis of NAFLD was not subjected to any semiquantitative indices. ${ }^{53}$ Ultrasonography is a well-established and cost-effective imaging technique for the diagnosis of hepatic steatosis, and is especially valuable for screening a large population at risk of NAFLD given that it is impossible to obtain the gold standard biopsy for large samples. ${ }^{55}$ Moreover, the severity of NAFLD is not stated clearly in the health check-up data so our results cannot be linked to NAFLD stage.

Acknowledgements We would like to thank all subjects who participated in the study and the staff at the Center for Health Management of Shandong Provincial QianFoShan Hospital and the Center for Health Management of Shandong Provincial Hospital.

Contributors SW and FX designed the study, carried out statistical analysis and wrote the manuscript. CZ, GZ, YL and HJ helped design the study, LD and $X S$ contributed to data analysis and $Z Y$ helped write the manuscript.

Funding This work was supported by two grants from the National Natural Science Fund (No. 81102191 and No. 81273082) of China.

Competing interests None declared.

Patient consent Obtained.

Ethics approval The Ethics Committee of the School of Public Health, Shandong University approved this study.

Provenance and peer review Not commissioned; externally peer reviewed.

Data sharing statement No additional data are available.
Open Access This is an Open Access article distributed in accordance with the Creative Commons Attribution Non Commercial (CC BY-NC 4.0) license, which permits others to distribute, remix, adapt, build upon this work noncommercially, and license their derivative works on different terms, provided the original work is properly cited and the use is non-commercial. See: http:// creativecommons.org/licenses/by-nc/4.0/

\section{REFERENCES}

1. Chalasani N, Younossi Z, Lavine JE, et al. The diagnosis and management of non-alcoholic fatty liver disease: Practice Guideline by the American Association for the Study of Liver Diseases, American College of Gastroenterology, and the American Gastroenterological Association. Hepatology 2012;55:2005-23.

2. Blachier M, Leleu H, Peck-Radosavljevic M, et al. The burden of liver disease in Europe: a review of available epidemiological data. $J$ Hepatol 2013;58:593-608.

3. Szczepaniak LS, Nurenberg P, Leonard D, et al. Magnetic resonance spectroscopy to measure hepatic triglyceride content: prevalence of hepatic steatosis in the general population. $\mathrm{Am}$ $J$ Physiol Endocrinol Metab 2005;288:E462-8.

4. Ratziu V, Bellentani $\mathrm{S}$, Cortez-Pinto $\mathrm{H}$, et al. A position statement on NAFLD/NASH based on the EASL 2009 special conference. $J$ Hepatol 2010;53:372-84.

5. Younossi ZM, Stepanova M, Afendy M, et al. Changes in the prevalence of the most common causes of chronic liver diseases in the United States from 1988 to 2008. Clin Gastroenterol Hepatol 2011;9:524-30.

6. Fan JG, Saibara T, Chitturi S, et al. What are the risk factors and settings for non-alcoholic fatty liver disease in Asia-Pacific? $J$ Gastroenterol Hepatol 2007;22:794-800.

7. Fan JG, Farrell GC. Epidemiology of non-alcoholic fatty liver disease in China. J Hepatol 2009;50:204-10.

8. Li Z, Xue J, Chen P, et al. Prevalence of nonalcoholic fatty liver disease in mainland of China: a meta-analysis of published studies. $J$ Gastroenterol Hepatol 2014;29:42-51.

9. Adams LA, Lymp JF, St Sauver J, et al. The natural history of nonalcoholic fatty liver disease: a population-based cohort study. Gastroenterology 2005;129:113-21.

10. McCullough AJ. The clinical features, diagnosis and natural history of nonalcoholic fatty liver disease. Clin Liver Dis 2004;8:521-33.

11. Asrih M, Jornayvaz FR. Inflammation as a potential link between nonalcoholic fatty liver disease and insulin resistance. J Endocrinol 2013;218:R25-36.

12. Hamaguchi M, Kojima T, Takeda N, et al. The metabolic syndrome as a predictor of nonalcoholic fatty liver disease. Ann Intern Med 2005;143:722-8.

13. Hamaguchi M, Kojima T, Takeda N, et al. Nonalcoholic fatty liver disease is a novel predictor of cardiovascular disease. World $J$ Gastroenterol 2007:13:1579-84.

14. Bae JC, Rhee EJ, Lee WY, et al. Combined effect of nonalcoholic fatty liver disease and impaired fasting glucose on the development of type 2 diabetes: a 4-year retrospective longitudinal study. Diabetes Care 2011;34:727-9.

15. Ahmed $\mathrm{MH}$, Barakat S, Almobarak $\mathrm{AO}$. Nonalcoholic fatty liver disease and cardiovascular disease: has the time come for cardiologists to be hepatologists? J Obes 2012;2012:483135

16. Kogiso T, Moriyoshi Y, Shimizu S, et al. High-sensitivity C-reactive protein as a serum predictor of nonalcoholic fatty liver disease based on the Akaike Information Criterion scoring system in the general Japanese population. J Gastroenterol 2009;44:313-21.

17. Kishimoto T. IL-6: from its discovery to clinical applications. Int Immunol 2010;22:347-52.

18. Tarantino G, Conca $P$, Pasanisi F, et al. Could inflammatory markers help diagnose nonalcoholic steatohepatitis? Eur J Gastroenterol Hepatol 2009;21:504-11.

19. Tarantino G, Savastano S, Capone D, et al. Spleen: a new role for an old player? World J Gastroenterol 2011;17:3776-84.

20. Lesmana CR, Hasan I, Budihusodo U, et al. Diagnostic value of a group of biochemical markers of liver fibrosis in patients with non-alcoholic steatohepatitis. J Dig Dis 2009;10:201-6.

21. Maleki I, Aminafshari MR, Taghvaei T, et al. Serum immunoglobulin A concentration is a reliable biomarker for liver fibrosis in non-alcoholic fatty liver disease. World J Gastroenterol 2014;20:12566-73.

22. Lee YJ, Lee HR, Shim JY, et al. Relationship between white blood cell count and nonalcoholic fatty liver disease. Dig Liver Dis 2010;42:888-94. 
23. Lorenzo C, Hanley AJ, Haffner SM. Differential white cell count and incident type 2 diabetes: the insulin resistance atherosclerosis study. Diabetologia 2014;57:83-92.

24. Lohsoonthorn V, Dhanamun B, Williams MA. Prevalence of metabolic syndrome and its relationship to white blood cell count in a population of Thai men and women receiving routine health examinations. Am J Hypertens 2006;19:339-45.

25. Zeng MD, Fan JG, Lu LG, et al. Guidelines for the diagnosis and treatment of nonalcoholic fatty liver diseases. J Dig Dis 2008;9:108-12.

26. Schunk D. A Markov chain Monte Carlo algorithm for multiple imputation in large surveys. AStA Adv Stat Anal 2008;92:101-14.

27. Das SK, Mukherjee S, Vasudevan DM, et al. Comparison of haematological parameters in patients with non-alcoholic fatty liver disease and alcoholic liver disease. Singapore Med J 2011;52:175-81.

28. Fargion S, Porzio M, Fracanzani AL. Nonalcoholic fatty liver disease and vascular disease: state-of-the-art. World J Gastroenterol 2014;20:13306-24.

29. Amarapurkar D, Kamani $\mathrm{P}$, Patel $\mathrm{N}$, et al. Prevalence of non-alcoholic fatty liver disease: population based study. Ann Hepatol 2007;6:161-3.

30. Frith J, Day CP, Henderson E, et al. Non-alcoholic fatty liver disease in older people. Gerontology 2009;55:607-13.

31. Vernon G, Baranova A, Younossi ZM. Systematic review: the epidemiology and natural history of non-alcoholic fatty liver disease and non-alcoholic steatohepatitis in adults. Aliment Pharmacol Ther 2011;34:274-85.

32. Chen ZW, Chen LY, Dai HL, et al. Relationship between alanine aminotransferase levels and metabolic syndrome in nonalcoholic fatty liver disease. J Zhejiang Univ Sci B 2008;9:616-22.

33. Caballería L, Pera G, Auladell MA, et al. Prevalence and factors associated with the presence of nonalcoholic fatty liver disease in an adult population in Spain. Eur J Gastroenterol Hepatol 2010;22:24-32

34. Liu Y, Dai M, Bi Y, et al. Active smoking, passive smoking, and risk of nonalcoholic fatty liver disease (NAFLD): a population-based study in China. J Epidemiol 2013;23:115-21.

35. Nseir W, Hellou E, Assy N. Role of diet and lifestyle changes in nonalcoholic fatty liver disease. World J Gastroenterol 2014;20:9338-44.

36. Hu X, Huang Y, Bao Z, et al. Prevalence and factors associated with nonalcoholic fatty liver disease in Shanghai work-units. BMC Gastroenterol 2012;12:123.

37. Angulo P. GI epidemiology: nonalcoholic fatty liver disease. Aliment Pharmacol Ther 2007;25:883-9.

38. Loomba R, Abraham M, Unalp A, et al. Association between diabetes, family history of diabetes, and risk of nonalcoholic steatohepatitis and fibrosis. Hepatology 2012;56:943-51.

39. Tarantino G, Finelli C. What about non-alcoholic fatty liver disease as a new criterion to define metabolic syndrome? World $\mathrm{J}$ Gastroenterol 2013;19:3375-84

40. Riquelme A, Arrese M, Soza A, et al. Nonalcoholic fatty liver disease and its association with obesity, insulin resistance and increased serum levels of C-reactive protein in Hispanics. Liver Int 2009;29:82-8.

41. Nigam $P$, Bhatt SP, Misra A, et al. Non-alcoholic fatty liver disease is closely associated with sub-clinical inflammation: a case-control study on Asian Indians in North India. PLoS ONE 2013;8:e49286.

42. Uchihara M, Izumi N. [High-sensitivity C-reactive protein (hs-CRP): a promising biomarker for the screening of non-alcoholic steatohepatitis (NASH)]. Nippon Rinsho 2006;64:1133-8.

43. Park SH, Kim BI, Yun JW, et al. Insulin resistance and C-reactive protein as independent risk factors for non-alcoholic fatty liver disease in non-obese Asian men. J Gastroenterol Hepatol 2004;19:694-8.

44. Das SK, Balakrishnan V. Role of cytokines in the pathogenesis of non-alcoholic fatty liver disease. Indian J Clin Biochem 2011;26:202-9.

45. Tasci I, Dogru T, Ercin CN, et al. Adipokines and cytokines in non-alcoholic fatty liver disease. Aliment Pharmacol Ther 2008;28:266-7.

46. Day CP, James OF. Steatohepatitis: a tale of two "hits"? Gastroenterology 1998;114:842-5.

47. Jimba S, Nakagami T, Takahashi M, et al. Prevalence of nonalcoholic fatty liver disease and its association with impaired glucose metabolism in Japanese adults. Diabet Med 2005;22:1141-5.

48. Chao TT, Hsieh CH, Lin JD, et al. Use of white blood cell counts to predict metabolic syndrome in the elderly: a 4 year longitudinal study. Aging Male 2014;17:230-7.

49. Gharipour M, Ramezani MA, Sadeghi M, et al. Sex based levels of C-reactive protein and white blood cell count in subjects with metabolic syndrome: Isfahan Healthy Heart Program. J Res Med Sci 2013;18:467-72.

50. Botezelli JD, Mora RF, Dalia RA, et al. Exercise counteracts fatty liver disease in rats fed on fructose-rich diet. Lipids Health Dis 2010;9:116.

51. Wolf AM, Busch B, Kuhlmann HW, et al. Histological changes in the liver of morbidly obese patients: correlation with metabolic parameters. Obes Surg 2005;15:228-37.

52. Pearson TA, Mensah GA, Alexander RW, et al. Markers of inflammation and cardiovascular disease: application to clinical and public health practice: a statement for healthcare professionals from the Centers for Disease Control and Prevention and the American Heart Association. Circulation 2003;107:499-511.

53. Ballestri S, Romagnoli D, Nascimbeni F, et al. Role of ultrasound in the diagnosis and treatment of nonalcoholic fatty liver disease and its complications. Expert Rev Gastroenterol Hepatol 2015;9:603-27.

54. Ballestri S, Lonardo A, Romagnoli D, et al. Ultrasonographic fatty liver indicator, a novel score which rules out NASH and is correlated with metabolic parameters in NAFLD. Liver Int 2012;32:1242-52.

55. Sinn DH, Gwak GY, Park HN, et al. Ultrasonographically detected non-alcoholic fatty liver disease is an independent predictor for identifying patients with insulin resistance in non-obese, non-diabetic middle-aged Asian adults. Am J Gastroenterol 2012;107:561-7. 